\title{
Three Dimensional Collagen Gels as a Cell Culture Matrix for the Study of Live Cells by Raman Spectroscopy
}

\author{
Franck Bonnier \\ Technological University Dublin, Franck.Bonnier@tudublin.ie \\ Aidan Meade \\ Technological University Dublin, aidan.meade@tudublin.ie \\ S. Merzha \\ Technological University Dublin
}

See next page for additional authors

Follow this and additional works at: https://arrow.tudublin.ie/nanolart

Part of the Nanoscience and Nanotechnology Commons

\section{Recommended Citation}

Bonnier, F. et al (2010) Three Dimensional Collagen Gels as a Cell Culture Matrix for the Study of Live Cells by Raman Spectroscopy. Analyst, 135, pp.1697-1703. doi:10.1039/c0an00060d

This Article is brought to you for free and open access by the NanoLab at ARROW@TU Dublin. It has been accepted for inclusion in Articles by an authorized administrator of ARROW@TU Dublin. For more information, please contact arrow.admin@tudublin.ie, aisling.coyne@tudublin.ie,gerard.connolly@tudublin.ie. Funder: National Biophotonics and Imaging Platform (NBIP) Ireland funded under the Higher Education Authority PRTLI (Programme for Research in Third Level Institutions) Cycle 4, co-funded by the Irish Government and the European Union.

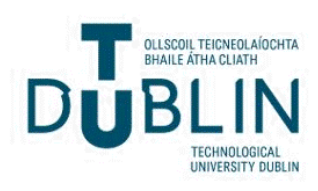




\section{Authors}

Franck Bonnier, Aidan Meade, S. Merzha, Peter Knief, Kunal Bhattacharya, Fiona Lyng, and Hugh Byrne 


\title{
Three dimensional collagen gels as a cell culture matrix for the study of live cells by Raman spectroscopy
}

\author{
F. Bonnier ${ }^{*}$, A.D. Meade, S. Merzha, P. Knief, K. Bhattacharya, \\ F.M. Lyng, H.J. Byrne
}

Focas Research Institute, Dublin Institute of Technology (DIT), Camden Row, Dublin 8, Ireland

\begin{abstract}
Three dimensional collagen gels are evaluated as matrices for the study of live cells by Raman spectroscopy. The study is conducted on a human lung adenocarcinoma (A549) and a spontaneously immortalized human epithelial keratinocyte (HaCaT) cell line. It is demonstrated, using the Alamar Blue assay, that both cell models exhibit enhanced viability in collagen matrices compared to quartz substrates, commonly used for Raman spectroscopy. Using principal component analysis, it is shown that the Raman spectral analysis of cells in collagen matrices are minimally contaminated by substrate contributions and the cell to cell spectral variations are greatly reduced compared to those measured on quartz substrates. Furthermore, the spectral measurements are seen to have little contribution from the cell culture medium, implying that cultures can be kept viable over prolonged measurement or mapping procedures.
\end{abstract}

Keywords: Raman Spectroscopy; Live cell imaging; 3D Collagen gels; Quartz substrates

\footnotetext{
* Corresponding author. Tel.: +353 1 4027917; Fax: +353 1 4027904;

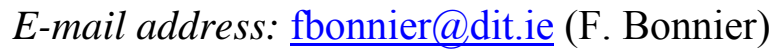




\section{Introduction}

Vibrational spectroscopic techniques are potential tools for the non-invasive, label free investigation of biological samples at a molecular level. Over the course of the last few years, the range of applications of Infrared and Raman spectroscopy has extended from intact tissue to single cell analysis ${ }^{1-4}$, elucidating different mechanisms involved in malignancy and cancer apparition as well as probing the efficiency of new drugs and the toxicity of nanoparticles at cellular level ${ }^{5-10}$. Infrared spectroscopic imaging has the advantage of speed of acquisition enabling rapid screening of large areas of tissue for diagnostic applications ${ }^{11,12}$. However, water is an extremely strong infrared absorber and thus applications in vivo are expected to be limited. Furthermore, the spatial resolution is diffraction limited to $\sim 10 \mu \mathrm{m}$, and thus IR absorption is not the favoured technique for live cell analysis. The weak scattering efficiency of water and the high spatial resolution of the visible or near -IR wavelengths employed for Raman microspectroscopy place this technique as the favoured tool for the study of living cells ${ }^{13,14}$, whereby live cells in a physiological solution can be mapped with an immersion lens giving sub cellular resolution of order of $1 \mu \mathrm{m}{ }^{15}$.

Although Raman spectroscopy is clearly a powerful tool for single cells analysis, many improvements have still to be realised to increase the quality of the information which can be gleaned. As with infrared spectroscopy, instrumental response, background and substrate response add to intrinsic sample variability ${ }^{16}$. Data analysis remains a difficulty and no standard protocol for data pre-processing has been established, emphasising the need for the minimisation of background contributions.

As the cellular and tissue material tends to be relatively thin, choice of substrate is critical, biocompatibility being a further consideration. Cells are commonly 
cultured in polystyrene petri dishes, but the Raman signal of the polymer itself is rich across the fingerprint region of interest $\left(400-1800 \mathrm{~cm}^{-1}\right){ }^{17}$ and, as the cells are thin and transparent, can completely swamp the cellular spectrum. The spectral features of glass are broad and overlap significantly with the region of interest of the cellular spectra ${ }^{18}$.

Quartz is commonly used as an alternative, as, although it contributes to the final spectra (fig.1 C and D), it does not contain any specific sharp peaks which interfere with the cellular spectrum and its contribution can be at least partially removed by a subtraction or division pre-processing procedure. Although they represent a workable solution, quartz windows are far from ideal, however. Substrate contributions will vary with sample density (transmittance) or indeed variations in focussing at the calibration or measurement stage, causing difficulties in standardisation of the background removal during the data pre-processing stage. Furthermore, quartz is not adapted for cell culture and can have toxic effects resulting in death or modification of the metabolism of cells. Improved biocompatibility of quartz for biospectroscopic applications can be achieved by the deposition of a thin layer of gelatin although spectral contributions from the substrate itself are still problematic ${ }^{19}$.

Confocal operation, available in some Raman microspectrometers, can reduce the spectral contribution of the substrate, as previously described by Puppels et al. for single cells ${ }^{20,21}$. Nevertheless, when performing measurements on optically thin single cells grown on a substrate, substrate contributions to the Raman spectra can still be significant. Furthermore, confocal operation is not available on many commercial spectrometers. 


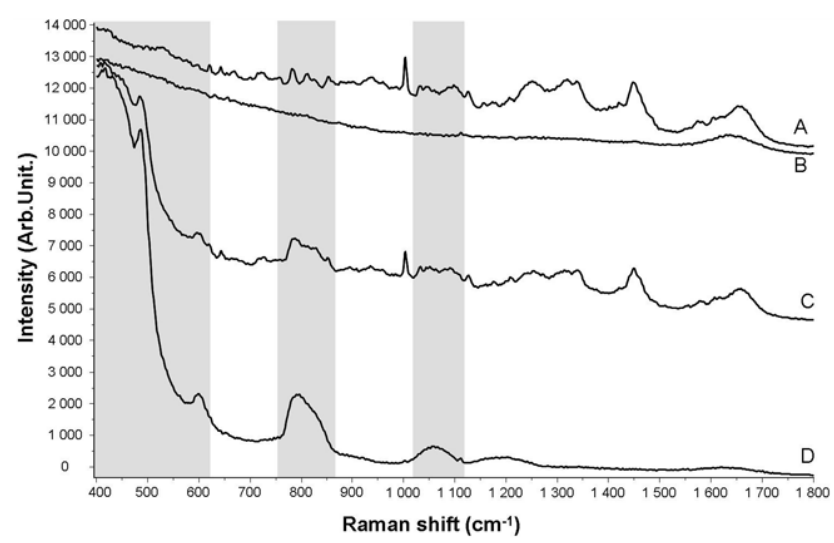

Fig. 1. Raman spectra recorded on different substrates. For each experiment an example of spectra recording from the cells is compared to the signal obtained from the corresponding substrate used (collagen gel or quartz). Spectra are offset for clarity. A: spectrum recorded from cells grown on collagen gel. B: background signal obtained from the Collagen gel. C: spectrum recorded from cells grown on quartz. D: background signal obtained from the quartz window.

As neither confocal operation nor spectral preprocessing are universally successful in eliminating substrate contributions, a routine alternative is desirable. This work aims to demonstrate the advantages of 3-dimensional collagen matrices as support matrices for the spectroscopic analysis of live cells, both in terms of reduced substrate spectral contributions (fig.1 A and B) as well as improved physiological environment of the cells. The extra-cellular matrix (ECM) encountered in-vivo consists of many different polymers, the most abundant of which is collagen type I. This fibrous protein is present in every compartment of the body and provides a 3D microenvironment for the cell. In vitro, different biomaterial surfaces can affect the cellular function, from adhesion and motility ${ }^{22,23}$ to proliferation and differentiation ${ }^{24}$ or gene expression ${ }^{25}$. Cellular functions in $2 \mathrm{D}$ and $3 \mathrm{D}$ systems are obviously different due to the limited interaction between cells and their microenvironment in $2 \mathrm{D}$ culture systems ${ }^{26,27}$. 3D collagen gels providing enhanced interaction with the 
surrounding collagenous network, are closer to the extra cellular matrix and mimic the morphology and phenotype of cells in vivo ${ }^{28}$.

\section{Materials and methods}

\subsection{A549 and HaCaT Cell lines}

A549 cells from a human lung adenocarcinoma with the alveolar type II phenotype were obtained from ATTC (Manassas, VA, USA). Cells were cultured in RPMI 1640 (Gibco, Karlsruhe) supplemented with L-glutamine, penicillin and streptomycin (Gibco) and 10\% foetal calf serum (FCS, Biochrom, Berlin) in a humidified atmosphere containing $5 \% \mathrm{CO}_{2}$ at $37^{\circ} \mathrm{C}$.

HaCaT cells are a spontaneously immortalized human epithelial keratinocyte cell line derived from adult skin ${ }^{29}$. They are aneuploid, non-tumourigenic and p53 mutated adult keratinocytes. HaCaT cells were cultured in Dulbecco's MEM: F12 (1:1) medium (Sigma, Dorset, U.K.) containing 10\% fetal calf serum (Gibco, Irvine, U.K.) $1 \%$ penicillin-streptomycin solution 1000 IU (Gibco, Irvine, U.K.), 2mM Lglutamine (Gibco, Irvine, UK) and $1 \mu \mathrm{g} / \mathrm{mL}$ hydrocortisone (Sigma, dorset,UK) in a humidified atmosphere containing $5 \% \mathrm{CO} 2$ at $37{ }^{\circ} \mathrm{C}$.

\subsection{Collagen gels}

Solutions of collagen I from rat tail tendons (Gibco) were used for preparation of the collagen gels. The $5 \mathrm{mg} / \mathrm{mL}$ solution was mixed with sterile $10 \mathrm{X}$ phosphate buffered saline (PBS), sterile distilled water $(\mathrm{dH} 2 \mathrm{O})$ and $1 \mathrm{M} \mathrm{NaOH}$. The appropriate relative quantities of these component is determined by the final concentration and volume 
needed. All the steps were carried out on ice to slow the gelation process. After mixing, the solution was placed in a petri-dish (for Raman spectroscopy) or 6 wellplate (for Alamar Blue assay analysis) before incubation at $37^{\circ} \mathrm{C}$ degrees in a $95 \%$ humidity incubator until a solid gel is formed (about 30mins).

\subsection{Sample preparation}

A549 and HaCaT cells were loaded at a concentration of $4 \times 10^{4}$ cells per substrate onto quartz windows (Hellma LTD., UK.), previously sterilised using ethanol then dried in a laminar flow, and collagen gels ( prepared as described previously). In order to fit with the different instruments, Petri dishes (30 mm diameter, Stardest, USA) have been preferred for Raman spectroscopic measurements and 6 well plates (Nunc A/S, USA) for the Alamar blue assays. All samples were incubated for $24 \mathrm{H}$ at $37^{\circ} \mathrm{C}$, 95\% $\mathrm{CO}_{2}$ before measurements.

\subsection{Raman spectroscopic measurements}

A Horiba Jobin-Yvon LabRAM HR800 spectrometer with an external $300 \mathrm{~mW}$ diode laser operating at $785 \mathrm{~nm}$ as source was used throughout this work. For all measurements, a x100 immersion objective (LUMPlanF1, Olympus) was employed which provided a spatial resolution of $\sim 1 \mu \mathrm{m}$ at the sample. The confocal hole was set at $100 \mu \mathrm{m}$ for all measurements, the specified setting for confocal operation. The system was pre-calibrated to the $520.7 \mathrm{~cm}^{-1}$ spectral line of Silicon. The Labram system is a confocal spectrometer that contains two interchangeable gratings (300 and 900 lines/mm respectively). In the following experiments the 300 lines/mm grating 
was used, which gave a spectral dispersion of around $1.5 \mathrm{~cm}^{-1}$ per pixel. The backscattered Raman signal was integrated for 30 seconds over the spectral ranges from 400 to $1800 \mathrm{~cm}^{-1}$ and accumulated 3 times to improve the signal to noise ratio. The detector used was a 16-bit dynamic range Peltier cooled CCD detector. Images of the sample were acquired using a video camera within the system.

Spectra were recorded from the cellular nucleus, with about 25 spectra recorded per substrate.

In the case of the studies of cells on the quartz substrates, after $24 \mathrm{hrs}$ incubation, many cells were visually observed to be of an unhealthy morphology compared to the control. These were not selected for measurement as the primary objective of the study was to illustrate the improved measurement platform rather than evaluate the capabilities of Raman for cytotoxicological analyses. The results presented therefore represent varying relative contributions of cellular and substrate/environmental spectra, rather than variations in cellular spectra.

A quartz and collagen reference spectrum was acquired for each individual quartz window and collagen gel used.

\subsection{Data Analysis}

The different data analysis steps were performed using Matlab (Mathworks, USA). Before statistical analysis, a Savitsky-Golay filter $\left(5^{\text {th }}\right.$ order, 7 points) was applied to smooth the spectra and the reference spectrum constituting the background signal was subtracted.

Principal component analysis (PCA) is a method of multivariate analysis broadly used with datasets of multiple dimensions ${ }^{30}$. It allows the reduction of the number of 
variables in a multidimensional dataset, although it retains most of the variation within the dataset. The order of the PCs denotes their importance to the dataset. $\mathrm{PC}_{1}$ describes the highest amount of variation, $\mathrm{PC}_{2}$ the second greatest and so on. Therefore, $\operatorname{var}\left(\mathrm{PC}_{1}\right) \geq \operatorname{var}\left(\mathrm{PC}_{2}\right) \geq \operatorname{var}\left(\mathrm{PC}_{\mathrm{p}}\right)$, where var $\left(\mathrm{PC}_{\mathrm{i}}\right)$ represents the variance of $\mathrm{PC}_{\mathrm{i}}$ in the considered data set. Generally, the 3 first components represent more than $90 \%$ of the variance. This statistical method was preferred for this study to highlight the variability existing in the spectral data set recording during the different experiments. The other advantage of this method is the observation of loadings which represent the variance for each variable (wavenumber) for a given PC. Analysing the loadings of a PC can give information about the source of the variability inside a data set, derived from variations in the molecular components contributing to the spectra.

\subsection{Alamar blue assay}

The Alamar blue $(\mathrm{AB})$ assay, consisting in the cell viability measurement ${ }^{31}$, was carried out according to manufacturer's instructions. Briefly, control media was removed; the cells were rinsed with $\mathrm{PBS}$ and $2 \mathrm{~mL}$ of an $\mathrm{AB}$ solution $(5 \%[\mathrm{v} / \mathrm{v}]$ solution of $\mathrm{AB}$ dye) prepared in fresh media (without FBS or supplements) was added to each well. Following a 3 hours incubation, $\mathrm{AB}$ fluorescence was quantified at the respective excitation and emission wavelength of 540 and $595 \mathrm{~nm}$ using a Tecan Genios microplate reader. The results were averaged over 3 different independent experiments $(n=3)$, with 3 replicates per experiment.

\section{Results and discussions}




\subsection{Cell viability in Collagen gel matrices}

The Alamar blue reagent is a cell viability and proliferation indicator based on the conversion of the non fluorescent dye resazurin to resofurin which is highly red fluorescent. The process is based on a redox reaction only occurring in metabolically active cells, thus the intensity of the fluorescence is proportional to the number of living cells. The viability of the A549 and HaCaT cells was tested after 24 hrs

incubation at $37^{\circ} \mathrm{C}$, to demonstrate the increase of viability using collagen gels. It was furthermore essential to ensure that the concentrations of collagen used for the study were adapted not only for Raman spectroscopy but also for improved viability of cells. The normalized $\mathrm{AB}$ fluorescence was calculated, where normalization was with respect to the $\mathrm{AB}$ fluorescence of the cells on plastic Petri dishes. Note that all three substrates were seeded with the same number of cells. For both cell lines, the use of collagen gels significantly increases the viability of the cells compared to quartz windows. In the case of the A549 cell line, an increase from $54 \%$ on quartz windows to $75 \%$ on collagen gel was observed, or a $39 \%$ increase in viability in collagen gels compared to the uncoated quartz substrates (Data not shown). The HaCaT cell line seemed to be more affected by the different substrates and an increase from $34 \%$ to $97 \%$ was observed, a $285 \%$ increase in viability in the collagen gels compared to the uncoated quartz substrates. For comparison, the \% increase in viability of $\mathrm{HaCaT}$ cells on fibronectin, gelatine and lamanin coated quartz substrates as measured by the Alamar Blue assay was observed to be $\sim 45,66$ and $92 \%$ respectively ${ }^{19}$. Clearly the increased viability affected by the 3-D collagen gel is significantly higher. Providing the cells a collagen network as a substrate clearly stimulates the cells compared to quartz windows. Quartz has a toxic effect on cells and this is probably due to the 
surface properties (charge, smoothness etc.) of the windows and their unsuitability for cell adhesion.

It was expected that both cell lines cultured on collagen gels would exhibit $100 \%$ viability but the A549 cell line seemed not so compatible with the collagen gels. The optimal $\mathrm{pH}$ for polymerisation is 7.0 , as quoted by the manufacturer. However, as recently described by Sung and $\mathrm{al}^{28}$, the thickness of the collagen fibres can influence the viability of the cells and a better viability was obtained when the $\mathrm{pH}$ of prepolymerized gel was 7.7 . This hypothesis can explain why a $100 \%$ viability was not reached for either cell line. Nevertheless it is difficult to understand the difference observed for the two cells lines. Further experiments to establish the optimal conditions for the preparation of collagen gels for each cell line are underway.

Nevertheless, the collagen gels significantly outperform the quartz windows providing a viability and therefore cell metabolism closer to that observed in cells cultured on plastic. A collagenous network thus eliminates the toxicity from the quartz windows and should improve the accuracy of spectroscopic studies based on for example the action of different drugs or cytotoxic agents. Indeed, using this 3D matrix, no additional toxicity from the substrate contributes to the results making the comparisons between the classic biological assays and the Raman spectral features measured on cells possible.

\subsection{Collagen gels as suitable substrates for Raman spectroscopy on living cells}

Typical cellular spectral features such as the amide I band at $1650 \mathrm{~cm}^{-1}$, the C-H at $1446 \mathrm{~cm}^{-1}$, the phenylalanine at $1004 \mathrm{~cm}^{-1}$ or the $\mathrm{C}-\mathrm{C}$ twist of tyrosine and 
phenylalanine at 642 and $621 \mathrm{~cm}^{-1}$ respectively can be identified in all samples, as shown in figure 2 . These peaks are only a few examples of features of the detailed spectra and a complete assignment can be easily found in the literature ${ }^{13,14,32-35}$.

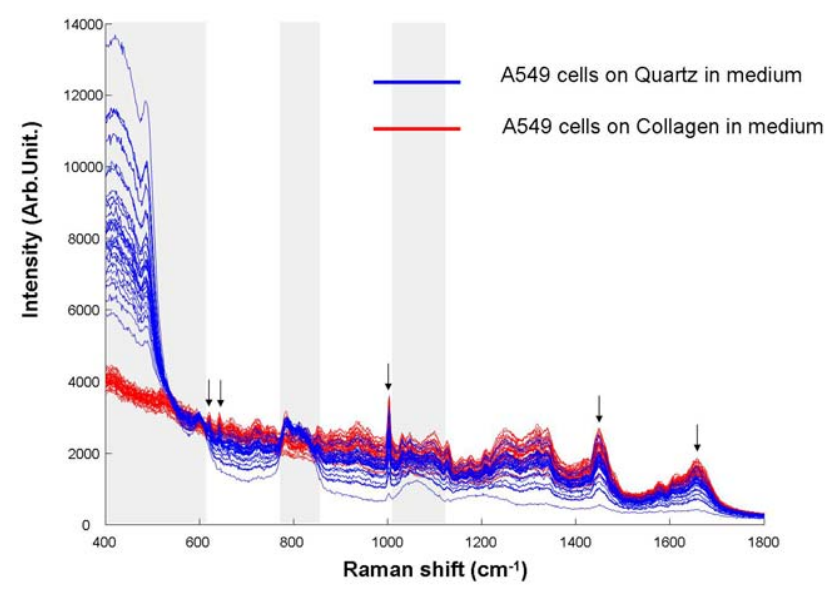

Fig. 2. Comparison of 2 Raman data sets recorded from cells grown on both quartz window and collagen gel in the spectral range $1800-400 \mathrm{~cm}^{-1}$. The 3 regions highlighted in grey are related to spectral windows influence by the contribution of the quartz. The black arrows indicate the most commonly peaks present in Raman spectra from cells.

Extensive pre-treatments and corrections (smoothing, baseline correction, normalisation, derivativisation, background subtraction, etc) are commonly applied to spectral datasets before data analysis. However, as this study was focused on the demonstration of suitability of collagen gels for Raman measurements of living cells rather than detail the biochemical content of the cells, it is more important to demonstrate the repeatability of the measurement in comparison to the commonly employed quartz standard. To this end, minimal preprocessing of the data was performed. All the statistical analyses have been performed after subtraction of the appropriate background signal recorded on the collagen or quartz windows, followed by a gentle smoothing (Savitzky-Golay $5^{\text {th }}$ order, 7 points) to reduce the noise of the 
spectra. No further preprocessing of the data set was performed in order to highlight the influence of the substrate on the reproducibility of the spectra. PCA was then applied to visualise the variation between the spectra.

Spectra were recorded both in the presence of cell culture medium or after being replaced by $\mathrm{NaCl}$. The medium is rich in vitamins and proteins which can contribute to the recorded spectra. This point is particularly important in the case of studies based on living cells. For example, a prolonged experimental schedule of two days measurement of live cells cannot be performed without control of the temperature and the $\mathrm{CO}_{2}$ level but equally not without an adequate medium for the cells. By comparing these conditions, the variations due to the presence of medium can be visualised.

The 3D plot comparing the four different conditions for the case of the A549 cell line is displayed in the figure 3. By focusing first on the difference between collagen gels and quartz windows, a clear discrimination can be seen between the two substrates. The spectra from cells on collagen gels are clustered in a quite compact group while the spectra recorded from cells on quartz windows are considerably more scattered. Although PC1 represents $76 \%$ of the variance of the four groups, it is noticeable that the spectra recorded on collagen gels have a negligible variance according to this PC. From the point of view of the presence of medium or $\mathrm{NaCl}$ solution during the measurement, no difference is discernible for spectra from cells on collagen as the two groups are well overlapped. The spectra from cells on quartz windows are however grouped, albeit loosely, according to whether they were performed in medium or a solution of $\mathrm{NaCl}$. Overall, the variance within spectra on quartz substrates is substantially higher than that for spectra on collagen gels. 


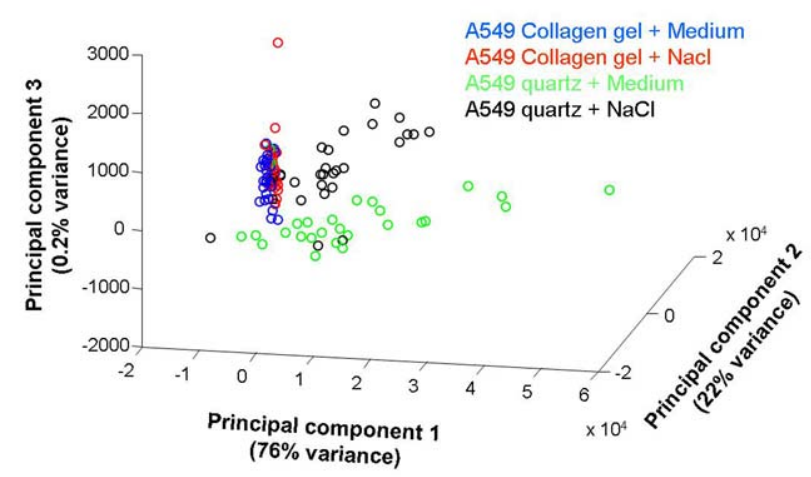

Fig. 3. 3D plot of the 3 first principal components after PCA performed on Raman spectra recorded from A549 cells grown on both collagen gels and quartz windows.

In terms of the effect of the medium on the spectral data set, it can be explained by the absorbance of the medium components by the collagen gel. In fact, the gels are a loose network formed by collagen fibres with considerable free space into which liquid can diffuse. This effect is apparent during sample preparation as the diffusion of the phenol red present in the medium through the collagen gel, results in the gel taking on a red/orange color after a few minutes. If the dye is able to permeate the gel, it is probable that other components of the medium can also, resulting in a reduced contribution of the medium to the spectral response.

The different loadings of the principal components, graphical representations of the variance according to the wavenumbers after PCA, are displayed in Figure 4.

Principal component 1 represents $76 \%$ of the variance of the complete dataset. In terms of the spectral features of the loadings, the most part of the variance corresponds to the regions influenced by the quartz signal. The strong similarity between the loading of PC1 and the quartz spectrum, especially in the range 400-850 $\mathrm{cm}^{-1}$, highlights the strong contribution of the quartz substrate during the recording of spectra. The fact that the 1 st principal component is dominated by the quartz signal confirms that confocal operation coupled with removal of the substrate contribution 
by subtraction is not sufficient. Given the dominance of quartz contributions to PC1, it is clear why spectra from cells in collagen gels have no variation in PC1 (Figure 3).
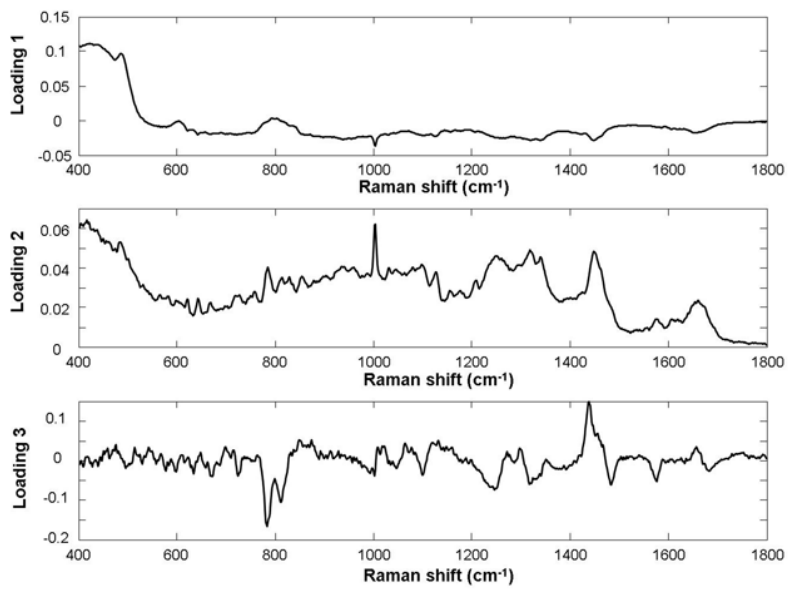

Fig. 4. Plot of the loadings corresponding to the 3 first principal components used for the plot of the figure 4.

Principal component 2 represents $22 \%$ of the variance and has a loading profile dominated by features from 800 to $1800 \mathrm{~cm}^{-1}$. The spectrum does not match well that of pure collagen (see figure 7) and is thus ascribed to variations in cellular features. Each of the spectra has been recorded on different live cells without any synchronisation before seeding. Although the nucleus of the cell was measured in all cases, the cells are obviously at different stages of the division process, involving heterogeneity in the morphology of the nucleus (condensation, number of chromosomes) having consequences on the uniformity of the spectral signature recorded..

The use of different cell lines is important to validate the observations and ensure that the results are not cell specific. HaCaT cells were grown on quartz in parallel and results were compared with those grown on collagen gels. Data preprocessing and PCA was conducted as was the case for the A549 cells. The 3D scatter plot obtained after the PCA analysis and the corresponding loadings. (Data not shown) are 
consistent with those for the A549 cell line. Once again, the spectra recording using the quartz substrate are considerably more scattered compared to those obtained from cells cultured on collagen gel. Principal component 1 represents $63 \%$ of the explained variance and is due to the contribution of quartz to the spectra. Principal component 2 represents $33 \%$ of the variance and in terms of the corresponding loading is related to cellular features rather than those of pure collagen. As was the case for the A549 cellline, no discrimination is visible between the spectra recorded using medium or $\mathrm{NaCl}$ with the collagen gels. Concerning the quartz windows, the discrimination is not as clear as previously seen with the A549 cell line and no clear distinction between the 2 conditions can be seen.

The comparisons using two different cell lines demonstrate that Collagen gels are a stable environment for sustaining live cells, and that Raman spectra of cells within the matrix are significantly less influenced by the substrate in comparison to quartz. Cellular spectra are observed to be considerably less varied and minimal contributions from the matrix are observable. Furthermore, the spectra recorded have minimal contributions from the cell culture medium which facilitates prolonged measurement or mapping in a viable environment. Such extended mapping periods are ideally required for full cellular and sub cellular analysis to evaluate for example mechanisms of interaction of nanoparticles or chemotherapeutic agents.

3.3. $Z$ profile of cells on quartz and collagen gels: effect of the focus during measurements

The spectra of cells recorded on quartz are scattered over the 3D PCA plot mainly due to variation of the quartz contributions and more precisely due to different 
positioning of the focus. The importance of the contribution of the substrate during the recording is related to the distance between the lens and the surface of the quartz during the measurement. To highlight this effect, 10 different $\mathrm{Z}$ profiles have been recorded on cells grown on quartz windows and collagen gels. The step between two $\mathrm{Z}$ positions was set at $1 \mu \mathrm{m}$ and spectra recorded from 0 to $+10 \mu \mathrm{m}$. The zero position corresponds to the focal point of the laser on the sample. Figure 5A shows the mean of six spectra extracted for each $\mathrm{Z}$ profile of cells on quartz and figure $5 \mathrm{~B}$ the mean of six spectra extracted at the same positions from the collagen gels. The shaded regions are the spectral windows with a contribution from the quartz. The decrease of the spectral feature related to the quartz according to the different $\mathrm{Z}$ positions is notable. In the case of the collagen gels, the mean spectra are more constant and no contribution from the collagen can be identified. As an example of the resultant improvements in spectral information, the shaded region of figure $5 \mathrm{~B}$ in the spectral range $500-550 \mathrm{~cm}^{-1}$ highlights the presence of a band related to the disulfide bonds of protein, which is obscured by the quartz contribution in the spectra of figure 5A.

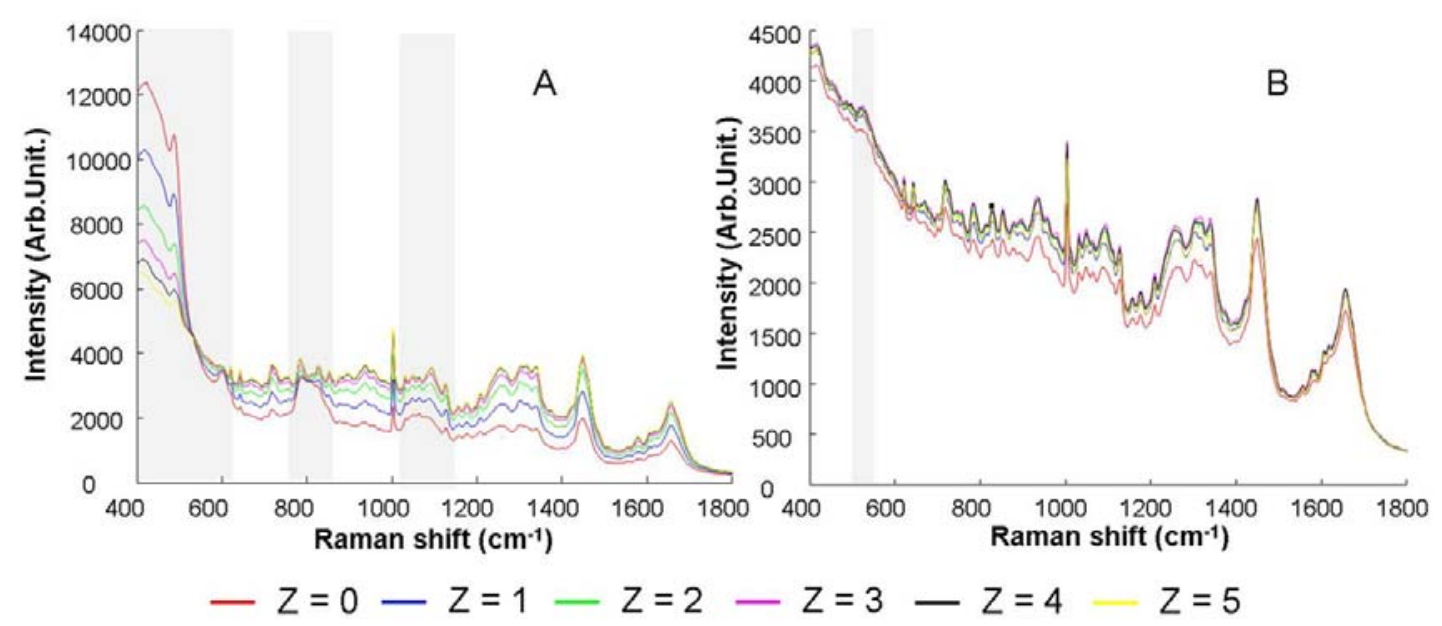

Fig. 5. Plot of the mean spectra after recording of Z profiles from HaCaT cells grown on both quartz and collagen gels in the range $1800-400 \mathrm{~cm}^{-1}$ from the positions $Z=0$ to $Z=5$. A: The spectral regions having a contribution from the quartz have been 
highlighted (grey). B: Without the use of the quartz as a substrate the region 500 and $550 \mathrm{~cm}^{-1}$ attributed to the disulfide bonds can be observed.

It is noted that little variation in the cellular spectrum is observed as a function of $\mathrm{Z}$ positioning. In the confocal mode, the $\mathrm{Z}$ sampling depth with the $\mathrm{x} 100$ objective is approximately $2 \mu \mathrm{m}$, and $\mathrm{Z}$ profiling over $5 \mu \mathrm{m}$ should be expected to differentiate nuclear and cytoplasmic contributions. Measurements were conducted 24hrs after seeding of the cells (on top of the 3-D gel), however, when they are still relatively flat and the cytoplasm overlying the nucleus is relatively thin. The spectrum of the considerably denser nucleus thus dominates. Differentiation of the regions as a function of focal positioning would be expected at longer times, when the cells have migrated further into the matrix.

Before performing the PCA analysis, for each $\mathrm{Z}$ position a corresponding background spectrum was subtracted from the spectra, to account for the systematically varied contribution. Figure 6 is the $3 \mathrm{D}$ plot of the spectra after PCA. The spectra corresponding to positions 1 to 5 recorded on collagen gels form a well clustered group while the spectra from the quartz windows are considerably dispersed in comparison. Although principal component 1 , which contains $78 \%$ of the variance, seems to be related to the cellular content (Data not shown), PC 2 is related to the quartz spectrum and accounts for $18 \%$ of the variance. By using adapted background spectra the most important variance is not due to the quartz anymore but it still appears strongly as the second principle component. It seems that the removal of the quartz contribution is more difficult that a simple subtraction and the most important point is that the spectra from collagen gel are naturally clustered after such corrections. 


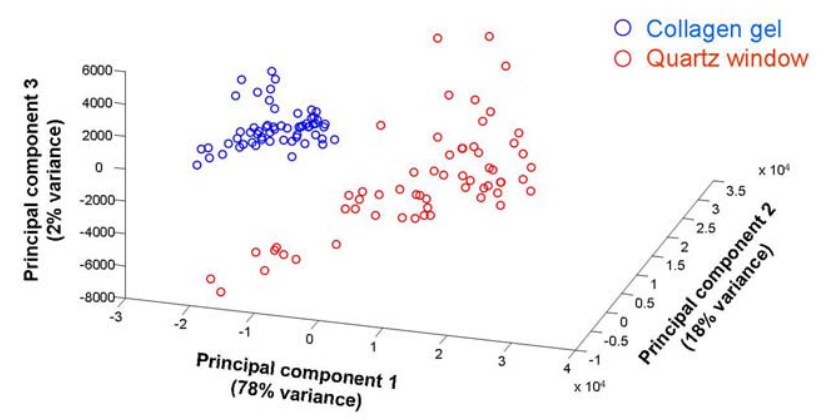

Fig. 6. 3D plot of the 3 first principal components after PCA performed on Raman spectra extracted from the $\mathrm{Z}$ profiles realised from $\mathrm{HaCaT}$ cells grown on both collagen gels and quartz windows. Only the spectra from the position $Z=0$ to $Z=5$ have been used for the plot.

\subsection{Why are spectra on collagen gel more constant?}

Because the spectra were recorded on cells not even synchronised according to cell cycle and by somewhat arbitrarily (manually) focusing on the nucleus, intrinsic cellular variations are expected in the data set. In the case of cells grown on quartz, however, the dominant variance $(78 \%)$ was from the substrate and it has been shown that this is in part due to the sensitivity to the z-focusing for the measurement. In the case of collagen gels, the dominant variance $(\sim 30 \%)$ was cellular in origin and little or no contribution from the substrate or indeed cell culture medium was observable. Representative spectra for both quartz substrates and collagen can be seen in figure 7A and 7B respectively. The spectrum from the quartz window has been expanded five fold in order to highlight the presence of spectral features in the region 1600$1700 \mathrm{~cm}^{-1}$ also present in the collagen gel spectra. The question remains as to the origin of the background contribution from collagen gels. The background spectra recorded are definitely not typical of raw collagen (figure 7B) or the plastic of the Petri dish and resemble more a broad baseline with no well defined peaks. One 
possibility is that the signal derives from an optical element of Raman spectrometer such as the immersion lens. Spectra were recorded using the lens "alone" (Data not shown) and the lens immersed in water (figure 7D). No spectrum was observed from the lens alone and therefore it appears that the background signal for the collagen gel is simply that of water. That implies that the density of the collagen fibres required to sustain cell growth is too low to give a signal in itself and that the only background contributions are from the water based solution used during the experiments $(\mathrm{NaCl}$ or medium) but also during the preparation of the gels. The vibrational spectrum of water is dominated by the symmetric and antisymmetric $\mathrm{O}-\mathrm{H}$ stretches in the region of $\sim 3400 \mathrm{~cm}^{-1}$. The bending modes at $\sim 1640 \mathrm{~cm}^{-1}$ are often overlooked as they are considerably weaker in comparison ${ }^{36,37}$. It is clear that the dominant background contribution to the Raman spectra of cells in collagen gels is simply the water in which the objective is immersed.

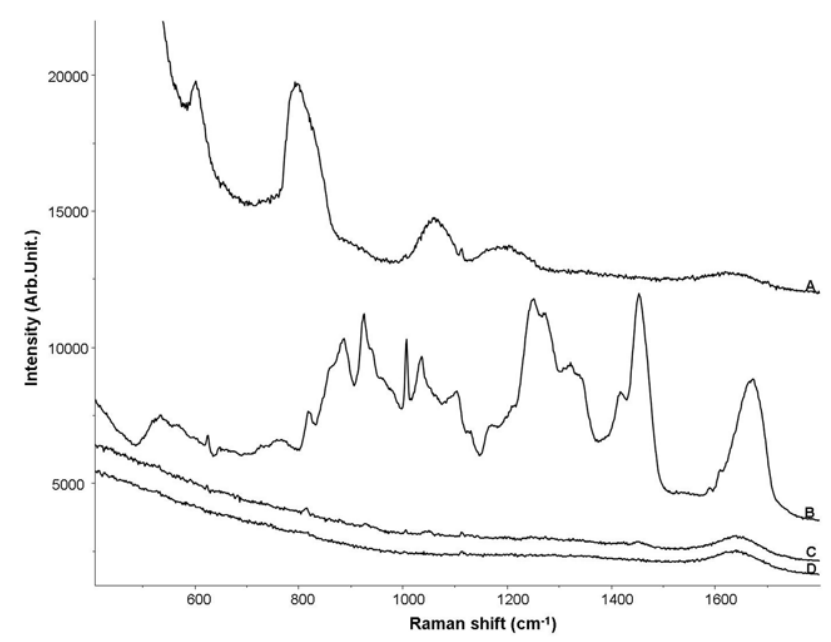

Fig. 7. Comparison of the signal recorded on the quartz, a pure sample of collagen, the collagen gel and water in the spectral range 1800-400 $\mathrm{cm}^{-1}$. A: The spectra of quartz have been expanded fivefold to increase the region $1500-1800 \mathrm{~cm}^{-1}$ and highlight the contribution of the water in the signal. B: The spectrum of pure collagen contains many specific peaks. C: spectrum recorded on the collagen gel D: Spectrum of water. 
For studies based on living cells it is necessary to perform measurements in medium or $\mathrm{NaCl}$ solution and the use of an immersion lens for Raman measurement is required. In these conditions it is not possible to get a weaker and more constant signal than the spectra of the water which does not contain any peaks overlapping with the signal from cells. The collagen gels are essentially invisible to Raman spectroscopy and appear like the perfect substrate for live cell studies.

\section{Conclusion}

During this study collagen gels have been compared to quartz windows as alternative substrates for live cell analysis using Raman spectroscopy. This preliminary study on the feasibility to record spectra from cells grown on collagen gels is really encouraging and opens up new perspectives. In comparison to quartz windows, cells on collagen gels show significantly higher viability. The spectra recorded are largely independent of the medium used implying a versatility which will facilitate extended periods of spectral mapping. Data analysis is considerably simplified due to the significantly reduced contribution from the substrate and the consistency of the signal obtained for the collagen gels will help to standardise the background removal from Raman data sets recorded from cells.

The benefits are observed to be applicable to the two cell lines studied, indicating that the benefits of collagen substrates are universal. Although this preliminary study has not identified effects of cell incubation time or optimised gel preparation conditions, the benefits of collagen gels as substrates for live cell analysis by Raman spectroscopy have clearly been demonstrated. 


\section{Acknowledgements}

This research was supported by the National Biophotonics and Imaging Platform (NBIP) Ireland funded under the Higher Education Authority PRTLI (Programme for Research in Third Level Institutions) Cycle 4, co-funded by the Irish Government and the European Union. 


\section{References}

1. R. A. Bitar, S. Martinho Hda, C. J. Tierra-Criollo, L. N. Zambelli Ramalho, M. M. Netto and A. A. Martin, Journal of biomedical optics, 2006, 11, 054001.

2. M. Diem, M. Romeo, S. Boydston-White, M. Miljkovic and C. Matthaus, The Analyst, 2004, 129, 880-885.

3. J. R. Mourant, R. R. Gibson, T. M. Johnson, S. Carpenter, K. W. Short, Y. R. Yamada and J. P. Freyer, Physics in medicine and biology, 2003, 48, 243-257.

4. K. W. Short, S. Carpenter, J. P. Freyer and J. R. Mourant, Biophysical journal, 2005, 88, 4274-4288.

5. N. S. Eikje, K. Aizawa and Y. Ozaki, Biotechnology annual review, 2005, 11, 191-225.

6. C. M. Krishna, G. Kegelaer, I. Adt, S. Rubin, V. B. Kartha, M. Manfait and G. D. Sockalingum, Biopolymers, 2006, 82, 462-470.

7. J. Ling, S. D. Weitman, M. A. Miller, R. V. Moore and A. C. Bovik, Applied optics, 2002, 41, 6006-6017.

8. J. R. Mourant, K. W. Short, S. Carpenter, N. Kunapareddy, L. Coburn, T. M. Powers and J. P. Freyer, Journal of biomedical optics, 2005, 10, 031106.

9. R. J. Swain and M. M. Stevens, Biochemical Society transactions, 2007, 35, 544-549.

10. P. Knief, C. Clarke, E. Herzog, M. Davoren, F. M. Lyng, A. D. Meade and H. J. Byrne, The Analyst, 2009, 134, 1182-1191.

11. F. Bonnier, D. Bertrand, S. Rubin, L. Venteo, M. Pluot, B. Baehrel, M. Manfait and G. D. Sockalingum, The Analyst, 2008, 133, 784-790.

12. A. Tfayli, O. Piot, A. Durlach, P. Bernard and M. Manfait, Biochimica et biophysica acta, 2005, 1724, 262-269.

13. I. Notingher, J. Selvakumaran and L. L. Hench, Biosensors \& bioelectronics, 2004, 20, 780-789.

14. R. J. Swain, G. Jell and M. M. Stevens, Journal of cellular biochemistry, 2008, 104, 1427-1438.

15. F. Draux, P. Jeannesson, A. Beljebbar, A. Tfayli, N. Fourre, M. Manfait, J. Sule-Suso and G. D. Sockalingum, The Analyst, 2009, 134, 542-548.

16. B. D. Beier and A. J. Berger, The Analyst, 2009, 134, 1198-1202.

17. B. J. Hunt and M. I. James, in Polymer Characterization, Blackie Press 1993,

18. E. Ó. Faoláin, Potential of vibrational spectroscopy in the diagnosis of human tumours (Thesis), Dublin Institute of Technology, Dublin 2006, http://arrow.dit.ie/sciendoc/25

19. A. D. Meade, F. M. Lyng, P. Knief and H. J. Byrne, Analytical and bioanalytical chemistry, 2007, 387, 1717-1728.

20. G. J. Puppels, F. F. de Mul, C. Otto, J. Greve, M. Robert-Nicoud, D. J. ArndtJovin and T. M. Jovin, Nature, 1990, 347, 301-303.

21. G. J. Puppels, W. Colier, J. H. F. Olminkhof, C. Otto, F. F. M. d. Mul and J. Greve, Journal of Raman Spectroscopy, 1991, 22, 217-225.

22. C. Gaudet, W. A. Marganski, S. Kim, C. T. Brown, V. Gunderia, M. Dembo and J. Y. Wong, Biophysical journal, 2003, 85, 3329-3335.

23. B. G. Keselowsky, D. M. Collard and A. J. Garcia, Biomaterials, 2004, 25, 5947-5954.

24. A. J. Garcia, M. D. Vega and D. Boettiger, Molecular biology of the cell, 1999, 10, 785-798. 
25. L. T. Allen, M. Tosetto, I. S. Miller, D. P. O'Connor, S. C. Penney, I. Lynch, A. K. Keenan, S. R. Pennington, K. A. Dawson and W. M. Gallagher, Biomaterials, 2006, 27, 3096-3108.

26. K. S. Smalley, M. Lioni and M. Herlyn, In vitro cellular \& developmental biology, 2006, 42, 242-247.

27. V. M. Weaver, O. W. Petersen, F. Wang, C. A. Larabell, P. Briand, C. Damsky and M. J. Bissell, The Journal of cell biology, 1997, 137, 231-245.

28. K. E. Sung, G. Su, C. Pehlke, S. M. Trier, K. W. Eliceiri, P. J. Keely, A. Friedl and D. J. Beebe, Biomaterials, 2009, doi:10.1016/j.biomaterials.2009.1005.1043.

29. P. Boukamp, S. Popp, K. Bleuel, E. Tomakidi, A. Burkle and N. E. Fusenig, Oncogene, 1999, 18, 5638-5645.

30. K. Varmuza, in Introduction to multivariate statistical analysis in chemometrics, ed.Taylor \& Francis Group, CRC Press, New york, 2009, ch.3, pp.59-102.

31. S. A. Ahmed, R. M. Gogal, Jr. and J. E. Walsh, Journal of immunological methods, 1994, 170, 211-224.

32. C. M. Krishna, G. D. Sockalingum, L. Venteo, R. A. Bhat, P. Kushtagi, M. Pluot and M. Manfait, Biopolymers, 2005, 79, 269-276.

33. A. Nijssen, T. C. Bakker Schut, F. Heule, P. J. Caspers, D. P. Hayes, M. H. Neumann and G. J. Puppels, The Journal of investigative dermatology, 2002, 119, 64-69.

34. I. Notingher and L. L. Hench, Expert review of medical devices, 2006, 3, $215-$ 234.

35. C. Yu, E. Gestl, K. Eckert, D. Allara and J. Irudayaraj, Cancer detection and prevention, 2006, 30, 515-522.

36. P. Piszczek, A. Grodzickia and B. Engelenb, Journal of Molecular Structure, 2003, 646, 45-54.

37. S. Venyaminov and F. G. Prendergast, Analytical biochemistry, 1997, 248, 234-245. 